\title{
Staircase diagrams and the enumeration of smooth Schubert varieties
}

\author{
Edward Richmond ${ }^{1} \|^{\mid}$and William Slofstra" \\ ${ }^{1}$ Department of Mathematics, Oklahoma State University, Stillwater, OK \\ ${ }^{2}$ Institute for Quantum Computing, University of Waterloo, Waterloo, ON
}

\begin{abstract}
In this extended abstract, we give a complete description and enumeration of smooth and rationally smooth Schubert varieties in finite type. In particular, we show that rationally smooth Schubert varieties are in bijection with a new combinatorial data structure called staircase diagrams.
\end{abstract}

Résumé. Dans ce résumé étendu, nous donnons une description complète et le dénombrement de variétés lisses et rationnellement lisses Schubert type fini. Dans particulier, nous montrons que les variétés de Schubert rationnellement lisses sont en bijection avec une nouvelle structure de données combinatoire appelé escalier diagrammes.

Keywords. Schubert varieties, rational smoothness, Billey-Postnikov decompositions

\section{Introduction}

Let $G$ be a simple Lie group over an algebraically closed field and fix a Borel subgroup $B \subseteq G$. The Schubert varieties $X(w)$ in the flag variety $G / B$ are indexed by the Weyl group $W$ of $G$. A natural question to ask is: when is $X(w)$ (rationally) smooth? Many different answers have been given to this question. For example, the Lakshmibai-Sandhya theorem states that a Schubert variety $X(w)$ of type $A$ is smooth if and only if the permutation $w$ avoids 3412 and 4231. There is an analogous pattern avoidance criteria for classical types due to Billey (1998), and a root-system pattern avoidance criteria for all finite types due to Billey and Postnikov (2005). A survey of several other characterizations can be found in Billey and Lakshmibai (2000).

Although these criteria allow us to efficiently recognize (rationally) smooth Schubert varieties, they do not allow us to enumerate such Schubert varieties. The main result of this paper, an extended abstract of Richmond and Slofstra(b), is the following theorem:

Theorem 1.1 If $W$ is a finite Weyl group, then there is a bijection between rationally smooth Schubert varieties $X(w)$ and "labelled" staircase diagrams.

\footnotetext{
${ }^{\dagger}$ Email: edward.richmondeokstate.edu

$\ddagger$ Email: weslofst@uwaterloo.ca

1365-8050 @ 2016 Discrete Mathematics and Theoretical Computer Science (DMTCS), Nancy, France
} 
Informally, a staircase diagram is a collection of connected "blocks" of vertices of a fixed graph, where the blocks are allowed to overlap each other, forming arrangements which resemble staircases with steps of irregular length, as shown in the pictures below.
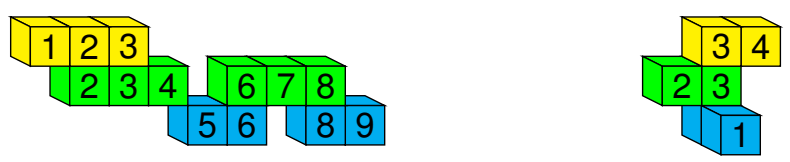

The precise definition of staircase diagram is given in Definition 3.1. Using Theorem 1.1, we can enumerate smooth and rationally smooth Schubert varieties in the finite classical types $A-D$. Specifically, define generating series

$$
A(t):=\sum_{n=0}^{\infty} a_{n} t^{n}, B(t):=\sum_{n=0}^{\infty} b_{n} t^{n}, C(t):=\sum_{n=0}^{\infty} c_{n} t^{n}, D(t):=\sum_{n=3}^{\infty} d_{n} t^{n}, B C(t):=\sum_{n=0}^{\infty} b c_{n} t^{n},
$$

where the coefficients $a_{n}, b_{n}, c_{n}, d_{n}$ denote the number of smooth Schubert varieties of types $A_{n}, B_{n}, C_{n}$ and $D_{n}$ respectively, and $b c_{n}$ denotes the number of rationally smooth Schubert varieties of type $B_{n}$. Since the Weyl groups of type $B_{n}$ and $C_{n}$ are isomorphic, and $X(w)$ is rationally smooth in type $B$ if and only if $X(w)$ is rationally smooth in type $C$, we refer to this last case as "type $B C$ ". For simply-laced types $A$ and $D$, Peterson's theorem states that a Schubert variety is rationally smooth if and only if it is smooth (Carrell and Kuttler (2003)), so these generating series cover all classes of smooth and rationally smooth Schubert varieties in finite classical type.

Theorem 1.2 Let $W(t):=\sum_{n} w_{n} t^{n}$ denote one of the above generating series, where $W=A, B, C$, $D$, or $B C$. Then

$$
W(t)=\frac{P_{W}(t)+Q_{W}(t) \sqrt{1-4 t}}{(1-t)^{2}\left(1-6 t+8 t^{2}-4 t^{3}\right)}
$$

where $P_{W}(t)$ and $Q_{W}(t)$ are polynomials given in Table 1

\begin{tabular}{ccc}
\hline Type & $P_{W}(t)$ & $Q_{W}(t)$ \\
\hline$A$ & $(1-4 t)(1-t)^{3}$ & $t(1-t)^{2}$ \\
$B$ & $\left(1-5 t+5 t^{2}\right)(1-t)^{3}$ & $\left(2 t-t^{2}\right)(1-t)^{3}$ \\
$C$ & $1-7 t+15 t^{2}-11 t^{3}-2 t^{4}+5 t^{5}$ & $t-t^{2}-t^{3}+3 t^{4}-t^{5}$ \\
$D$ & $\left(-4 t+19 t^{2}+8 t^{3}-30 t^{4}+16 t^{5}\right)(1-t)^{2}$ & $\left(4 t-15 t^{2}+11 t^{3}-2 t^{5}\right)(1-t)$ \\
$B C$ & $1-8 t+23 t^{2}-29 t^{3}+14 t^{4}$ & $2 t-6 t^{2}+7 t^{3}-2 t^{4}$ \\
\hline
\end{tabular}

Tab. 1: Polynomials in Theorem 1.2

In type $A$ the generating series for the number of smooth Schubert varieties is due to Haiman (Haiman. Bóna (1998), Bousquet-Mélou and Butler (2007)). For the other types this result is new. Table 2 gives the number of smooth and rationally smooth Schubert varieties in each type for rank $n \leq 6$. It is well known that the growth of the coefficients of a generating series is controlled by the singularity of smallest 


\begin{tabular}{cccccc}
\hline & $a_{n}$ & $b_{n}$ & $c_{n}$ & $d_{n}$ & $b c_{n}$ \\
\hline$n=1$ & 2 & 2 & 2 & & 2 \\
$n=2$ & 6 & 7 & 7 & & 8 \\
$n=3$ & 22 & 28 & 28 & 22 & 34 \\
$n=4$ & 88 & 116 & 114 & 108 & 142 \\
$n=5$ & 366 & 490 & 472 & 490 & 596 \\
$n=6$ & 1552 & 2094 & 1988 & 2164 & 2530 \\
\hline
\end{tabular}

Tab. 2: Number of smooth and rationally smooth Schubert varieties in ranks $n \leq 6$. By convention, $A_{1}=B_{1}=C_{1}$, and $D_{3}=A_{3}$.

modulus (Flajolet and Sedgewick, 2009, Theorem IV.7). For each generating series $W(t)$ in Theorem 1.2 . the smallest singularity is the root

$$
\alpha:=\frac{1}{6}(4-\sqrt[3]{17+3 \sqrt{33}}+\sqrt[3]{-17+3 \sqrt{33}}) \approx 0.228155
$$

of the polynomial $1-6 t+8 t^{2}-4 t^{3}$ appearing in the denominator. Thus we get an asymptotic formula for $w_{n}$ as an immediate corollary of Theorem 1.2 and (Flajolet and Sedgewick, 2009, Theorem IV.10).

Corollary 1.3 Let $W(t)=\sum w_{n} t^{n}$, where $W=A, B, C, D$, or BC. Then

$$
w_{n} \sim \frac{W_{\alpha}}{\alpha^{n+1}},
$$

where $W_{\alpha}:=\lim _{t \rightarrow \alpha}(\alpha-t) W(t)$. In particular, the number of (rationally) smooth Schubert varieties $w_{n}$ grows at the same rate for every finite classical Lie type. Table 3 gives the approximate value of $W_{\alpha}$ in each type.

\begin{tabular}{cccccc}
\hline & $A$ & $B$ & $C$ & $D$ & $B C$ \\
\hline$W_{\alpha}$ & 0.045352 & 0.062022 & 0.057301 & 0.067269 & 0.073972 \\
\hline
\end{tabular}

Tab. 3: Initial constant for the asymptotic number of Schubert varieties by type.

The main focus of this abstract is to explain the notion of staircase diagram and its connections with Coxeter groups. Due to the limited space, we only give a brief outline of the proof of Theorem 1.1 for simply laced Weyl groups. In this case, the adjective "labelled" can be dropped from Theorem 1.1 and we are free to concentrate on staircase diagrams without any additional structure. We also give an overview of the proof of Theorem 1.2, focusing on the simplest case, type $A$.

\section{Billey-Postnikov decompositions}

Before defining staircase diagrams, it is helpful to give some motivation. Let $(W, S)$ be a Coxeter system. Given a subset $J \subset S$, let $W_{J}$ be the parabolic subgroup generated by $J$. Let $W^{J}$ denote the set of minimal length left coset representatives of $W / W_{J}$. Every element $w \in W$ can be written uniquely as 
$w=v u$ for some $v \in W^{J}$ and $u \in W_{J}$. This is called the parabolic decomposition of $w$ with respect to $J$. We also let $D_{L}(w)$ and $D_{R}(w)$ denote the left and right descent sets of $w$ respectively, and $S(w)$ be the support of $w$, or in other words the set of simple reflections which appear in some reduced expression for $w$. Given an element $w \in W$, we say that a parabolic decomposition $w=v u, v \in W^{J}, u \in W_{J}$, is a Billey-Postnikov (BP) decomposition if $S(v) \cap J \subseteq D_{L}(u)$.

Theorem 2.1 (Richmond and Slofstra (a)) Let $w=v u$ be a parabolic decomposition of $w$ with respect to $J \subset S$. Then the following are equivalent:

- $w=v u$ is a BP decomposition.

- If $P_{J}$ is the parabolic subgroup of $G$ corresponding to $J$, and $X^{J}(v)$ is the Schubert variety in $G / P_{J}$ indexed by $v$, then $X(w)$ is a fibre bundle over $X^{J}(v)$ with fibre $X(u)$.

Consequently if $w=v u$ is a $B P$ decomposition with respect to $J$, then $X(w)$ is (rationally) smooth if and only if $X(u)$ and $X^{J}(v)$ are (rationally) smooth.

A parabolic decomposition (resp. BP decomposition) $w=v u$ with respect to $J$ is Grassmannian if $J=S \backslash\{s\}$ and $v \neq e$. Moreover, we say $w \in W$ is (rationally) smooth if $X(w)$ is (rationally) smooth.

Theorem $2.2($ Richmond and Slofstra (a)) Let $W$ be a finite Weyl group. An element $w \in W$ is rationally smooth if and only if we can write $w=v_{k} \cdots v_{1}$, where $v_{i}\left(v_{i-1} \cdots v_{1}\right)$ is a Grassmannian BP decomposition with respect to some $J_{i}$ and $X^{J_{i}}\left(v_{i}\right)$ is rationally smooth for each $i$.

Theorem 2.3 (Richmond and Slofstra (a)) Let $W$ be a finite Weyl group. Let $J=S \backslash\{s\}$ and suppose $v \in W^{J}$. Then the Grassmannian Schubert variety $X^{J}(v)$ is rationally smooth if and only if either:

- $v$ is a maximal element in $W_{S(v)}^{J \cap S(v)}$,

- $v$ is one of six infinite families in types $B_{n}$ or $C_{n}$, or $v$ is one of ten elements in types $F_{4}$ or $G_{2}$.

In particular, if $W$ is simply-laced and $X^{J}(v)$ is rationally smooth, then $v$ must be maximal in $W_{S(v)}^{J \cap S(v)}$.

These theorems give a way to generate all (rationally) smooth elements of a finite Weyl group. Specifically, we can build all such elements as iterated BP decompositions $v_{k} \cdots v_{1}$, where each $v_{i}$ is one of the elements listed in Theorem 2.3 However, two issues arise. First, given an iterated BP decomposition $v_{k-1} \cdots v_{1}$, we would like to know which elements $v_{k}$ listed in Theorem 2.3 give a BP decomposition $v_{k}\left(v_{k-1} \cdots v_{1}\right)$. Second, a rationally smooth element $w$ might have more than one iterated BP decomposition; we would like to generate rationally smooth elements bijectively. To resolve these issues, we introduce the notion of a staircase diagram.

\section{Staircase diagrams on graphs}

The rigorous definition of a staircase diagram requires some standard terminology concerning posets and graphs. Specifically, if $(X, \preceq)$ is a poset, recall that $x^{\prime} \in X$ covers $x \in X$ if $x^{\prime} \succ x$ and there is no $y \in X$ with $x^{\prime} \succ y \succ x$. A subset $Y \subset X$ is a chain if it is totally ordered, and saturated if $x^{\prime} \succ y \succ x$ for some $x^{\prime}, x \in Y$ implies that $y \in Y$. Given $A, B \subseteq X$, we say that $A \prec B$ if $a \prec b$ for all $a \in A, b \in B$. Since we will eventually be working with Coxeter-Dynkin diagrams, we use $S$ to denote the vertex set of a graph $\Gamma$, which we fix for this section. Given $s, t \in S$, we write $s$ adj $t$ to mean that $s$ is adjacent to $t$, or in other 
words that there is an edge between $s$ and $t$ in $\Gamma$. We allow $\Gamma$ to have multiple edges between two vertices, since Coxeter-Dynkin diagrams can have this property. However, the staircase diagrams only depend on whether $s$ and $t$ are adjacent (and since we focus on simply-laced Coxeter groups, edge multiplicities are not important). Throughout the paper, we assume that $\Gamma$ does not have any loops. We say that a subset $B \subset S$ is connected if the induced subgraph with vertex set $B$ is connected, and that $B, B^{\prime} \subset S$ are adjacent if some element of $B$ is adjacent to some element of $B^{\prime}$. Finally, given a collection $\mathcal{D} \subseteq 2^{S}$ and a vertex $s \in S$, we let

$$
\mathcal{D}_{s}:=\{B \in \mathcal{D} \mid s \in B\}
$$

Definition 3.1 Let $\mathcal{D}=(\mathcal{D}, \preceq)$ be a partially ordered subset of $2^{S}$ not containing the empty set. We say that $\mathcal{D}$ is a staircase diagram if the following are true:

1. Every $B \in \mathcal{D}$ is connected, and if $B$ covers $B^{\prime}$ then $B \cup B^{\prime}$ is connected.

2. The subset $\mathcal{D}_{s}$ is a chain for every $s \in S$.

3. If $s$ adj then $\mathcal{D}_{s} \cup \mathcal{D}_{t}$ is a chain, and $\mathcal{D}_{s}$ and $\mathcal{D}_{t}$ are saturated subchains of $\mathcal{D}_{s} \cup \mathcal{D}_{t}$.

4. If $B \in \mathcal{D}$, then there is some $s \in S$ (resp. $s^{\prime} \in S$ ) such that $B$ is the minimum element of $\mathcal{D}_{s}$ (resp. maximum element of $\mathcal{D}_{s^{\prime}}$ ).

This definition is meant to formalize an arrangement of blocks sitting over a graph, such that the blocks overlap each other in a particular way. Note that elements of the set $\mathcal{D}$ are called blocks. We now consider some specific examples illustrating the different parts Definition 3.1 .

Example 3.2 The picture

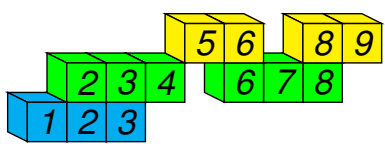

represents a staircase diagram $\mathcal{D}$ over a simple path with vertices $S=\left\{s_{1}, \ldots, s_{9}\right\}$, where $s_{i}$ is adjacent to $s_{i+1}$. The elements of $\mathcal{D}$ correspond to connected blocks of uniform color in this diagram (for notational simplicity, we pictorially label $s_{i}$ by $i$ ), so

$$
\mathcal{D}=\left\{\left\{s_{1}, s_{2}, s_{3}\right\},\left\{s_{2}, s_{3}, s_{4}\right\},\left\{s_{5}, s_{6}\right\},\left\{s_{6}, s_{7}, s_{8}\right\},\left\{s_{8}, s_{9}\right\}\right\} .
$$

The covering relations for $\mathcal{D}$ are given by the vertical adjacencies. In this case, $\mathcal{D}$ has covering relations

$$
\left\{s_{1}, s_{2}, s_{3}\right\} \prec\left\{s_{2}, s_{3}, s_{4}\right\} \prec\left\{s_{5}, s_{6}\right\} \text { and }\left\{s_{6}, s_{7}, s_{8}\right\} \prec\left\{s_{5}, s_{6}\right\} \text { and }\left\{s_{6}, s_{7}, s_{8}\right\} \prec\left\{s_{8}, s_{9}\right\} \text {. }
$$

Example 3.3 Let $\Gamma$ be the graph

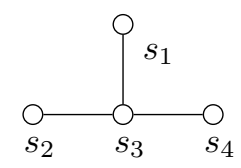

and consider the diagrams

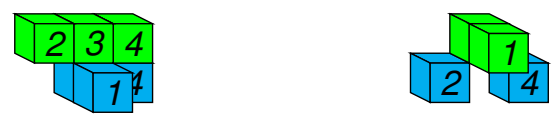


The first staircase diagram is $\left\{\left\{s_{1}, s_{3}, s_{4}\right\},\left\{s_{2}, s_{3}, s_{4}\right\}\right\}$ with $\left\{s_{1}, s_{3}, s_{4}\right\} \prec\left\{s_{2}, s_{3}, s_{4}\right\}$ and the second diagram is $\left\{\left\{s_{2}\right\},\left\{s_{4}\right\},\left\{s_{1}, s_{3}\right\}\right\}$ with covering relations $\left\{s_{2}\right\} \prec\left\{s_{1}, s_{3}\right\}$ and $\left\{s_{4}\right\} \prec\left\{s_{1}, s_{3}\right\}$.

Part (1) of Definition 3.1 states that the block of a diagram must be a connected subset of the vertices, and that blocks can only touch if they contain common or adjacent vertices. Part (2) of the definition states that blocks with a common vertex must be comparable, or in other words must be stacked one over the other. The following example show violations of parts (3) and (4) of Definition 3.1

Example 3.4 Consider the diagrams:
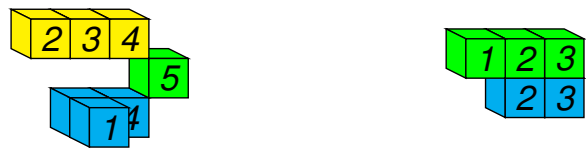

The first diagram violates part (3) of Definition 3.1 since the chain $\mathcal{D}_{s_{4}}$ is not a saturated subchain of $\mathcal{D}_{s_{4}} \cup \mathcal{D}_{s_{5}}$. The second diagram violates part (4) of Definition 3.1 since the block $\left\{s_{2}, s_{3}\right\}$ is not maximal in $\mathcal{D}_{s_{2}}=\mathcal{D}_{s_{3}}$.

Given $J \subset S$, we define $\mathcal{D}_{J}:=\{B \in \mathcal{D} \mid J \subseteq B\}$. By definition, $\mathcal{D}_{J}$ will be a chain for every $J$. Also as consequences of the definition, no block of $\mathcal{D}$ is a subset of any other block, and if two blocks contain a common element or are adjacent, then $B$ and $B^{\prime}$ are comparable in the partial order on $\mathcal{D}$.

Definition 3.1 is symmetric with respect to reversing the partial order $\preceq$ on $\mathcal{D}$, so we can make the following definition:

Definition 3.5 If $\mathcal{D}$ is a staircase diagram, then flip $(\mathcal{D})$ is the staircase diagram with the reverse partial order.

To get the pictorial diagram for $\operatorname{flip}(\mathcal{D})$, we simply flip the diagram from top to bottom. If $\mathcal{D}$ is the diagram in Example 3.2 then $\operatorname{flip}(\mathcal{D})$ is the first diagram given in the introduction. We finish the section with the following definition:

Definition 3.6 The support of a staircase diagram $\mathcal{D}$ is the set of vertices

$$
\mathrm{S}(\mathcal{D}):=\bigcup_{B \in \mathcal{D}} B
$$

We say $\mathcal{D}$ is connected if the support is a connected subset of the base graph. A subset $\mathcal{D}^{\prime} \subset \mathcal{D}$ is a subdiagram if $\mathcal{D}^{\prime}$ is a saturated subset of $\mathcal{D}$.

It is easy to see that a subdiagram of a staircase diagram with the induced partial order is a staircase diagram in its own right. Every staircase diagram $\mathcal{D}$ is a union of connected subdiagrams supported on the connected components of $\mathrm{S}(\mathcal{D})$.

\section{Staircase diagrams on Coxeter systems}

Let $(W, S)$ be a Coxeter system, and regard $S$ as the vertex set of the Coxeter-Dynkin graph. We say that a subset $J \subseteq S$ is spherical if the parabolic subgroup $W_{J}$ is finite.

Definition 4.1 We say that a staircase diagram $\mathcal{D}$ is spherical if every $B \in \mathcal{D}$ is spherical. 
Staircase diagrams can be used to describe iterated Billey-Postnikov decompositions. Specifically, given a staircase diagram $\mathcal{D}$ on a Coxeter system $(W, S)$, define functions $J_{R}, J_{L}: \mathcal{D} \rightarrow S$ by

$$
\begin{aligned}
& J_{R}(B) \cong J_{R}(B, \mathcal{D}):=\left\{s \in B \mid B \neq \min \left\{\mathcal{D}_{s}\right\}\right\} \text { and } \\
& J_{L}(B) \cong J_{L}(B, \mathcal{D}):=\left\{s \in B \mid B \neq \max \left\{\mathcal{D}_{s}\right\}\right\}=J_{R}(B, \operatorname{flip}(\mathcal{D})) .
\end{aligned}
$$

Definition 4.2 Given a spherical staircase diagram $\mathcal{D}$ on a Coxeter-Dynkin diagram, let $\bar{\lambda}(B)$ be the maximal element of $W_{B}^{J_{R}(B)}$, and set

$$
\Lambda(\mathcal{D}):=\bar{\lambda}\left(B_{n}\right) \bar{\lambda}\left(B_{n-1}\right) \cdots \bar{\lambda}\left(B_{1}\right) \in W,
$$

where $B_{1}, \ldots, B_{n}$ is some linear extension of the poset $\mathcal{D}$.

If $B$ and $B^{\prime}$ are incomparable in $\mathcal{D}$, then $B, B^{\prime}$ are disjoint and non-adjacent by Definition 3.1. In particular, $\bar{\lambda}(B)$ and $\bar{\lambda}\left(B^{\prime}\right)$ commute and thus $\Lambda(\mathcal{D})$ does not depend on the choice of linear extension. Also,

$$
J_{R}\left(B_{i}\right)=B_{i} \cap\left(B_{i-1} \cup \cdots \cup B_{1}\right)
$$

for every $i=1, \ldots, n$, and by definition $\bar{\lambda}\left(B_{i}\right) \in W^{J_{R}\left(B_{i}\right)}$, so the product in equation 11 is reduced in the sense that

$$
\ell(\Lambda(\mathcal{D}))=\ell\left(\bar{\lambda}\left(B_{n}\right)\right)+\cdots+\ell\left(\bar{\lambda}\left(B_{1}\right)\right) .
$$

Moreover, we have that $S(\Lambda(\mathcal{D}))=\mathrm{S}(\mathcal{D})$.

Example 4.3 The Coxeter-Dynkin diagram of $A_{n}$ is the simple path of length $n$, with vertex set $s_{1}, \ldots, s_{n}$.

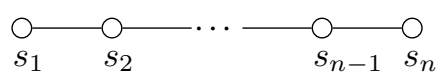

The staircase diagram $\mathcal{D}$ in Example 3.2 can be considered as a staircase diagram over the Coxeter group $A_{9}$. This diagram has a linear extension

$$
\left\{s_{1}, s_{2}, s_{3}\right\},\left\{s_{2}, s_{3}, s_{4}\right\},\left\{s_{6}, s_{7}, s_{8}\right\},\left\{s_{5}, s_{6}\right\},\left\{s_{8}, s_{9}\right\}
$$

with

$$
\begin{gathered}
J_{R}\left(\left\{s_{2}, s_{3}, s_{4}\right\}\right)=\left\{s_{2}, s_{3}\right\}, \quad J_{R}\left(\left\{s_{5}, s_{6}\right\}\right)=\left\{s_{6}\right\}, \quad J_{R}\left(\left\{s_{8}, s_{9}\right\}\right)=\left\{s_{8}\right\} \\
\text { and } J_{R}\left(\left\{s_{1}, s_{2}, s_{3}\right\}\right)=J_{R}\left(\left\{s_{6}, s_{7}, s_{8}\right\}\right)=\emptyset
\end{gathered}
$$

Thus

$$
\begin{aligned}
& \bar{\lambda}\left(\left\{s_{2}, s_{3}, s_{4}\right\}\right)=s_{2} s_{3} s_{4}, \quad \bar{\lambda}\left(\left\{s_{5}, s_{6}\right\}\right)=s_{6} s_{5}, \quad \bar{\lambda}\left(\left\{s_{8}, s_{9}\right\}\right)=s_{8} s_{9}, \\
& \bar{\lambda}\left(\left\{s_{1}, s_{2}, s_{3}\right\}\right)=s_{1} s_{2} s_{3} s_{1} s_{2} s_{1} \quad \text { and } \quad \bar{\lambda}\left(\left\{s_{6}, s_{7}, s_{8}\right\}\right)=s_{6} s_{7} s_{8} s_{6} s_{7} s_{6}
\end{aligned}
$$

and

$$
\Lambda(\mathcal{D})=\left(s_{8} s_{9}\right)\left(s_{6} s_{5}\right)\left(s_{6} s_{7} s_{8} s_{6} s_{7} s_{6}\right)\left(s_{2} s_{3} s_{4}\right)\left(s_{1} s_{2} s_{3} s_{1} s_{2} s_{1}\right)
$$

The main theorem of this section states that we can recover information about $\Lambda(\mathcal{D})$ from the diagram $\mathcal{D}$ alone. 
Theorem 4.4 Let $\mathcal{D}$ be a spherical staircase diagram. Then:

1. $\Lambda(\operatorname{flip}(\mathcal{D}))=\Lambda(\mathcal{D})^{-1}$.

2. $D_{R}(\Lambda(\mathcal{D}))=\left\{s \in S \mid \min \left(\mathcal{D}_{s}\right) \preceq \min \left(\mathcal{D}_{t}\right)\right.$ for all $s$ adj $\left.t\right\}$.

3. $D_{L}(\Lambda(\mathcal{D}))=D_{L}(\mathcal{D}):=\left\{s \in S \mid \max \left(\mathcal{D}_{s}\right) \succeq \max \left(\mathcal{D}_{t}\right)\right.$ for all $s$ adj $\left.t\right\}$.

4. Suppose $\Lambda(\mathcal{D})=v u$ is the parabolic decomposition with respect to a subset $J \subset S$. Then

$$
S(v)=\bigcup_{\substack{s \notin J \\ s \succeq \min \left(\mathcal{D}_{s}\right)}} B .
$$

With Theorem 4.4 we can make a connection between staircase diagrams and BP decompositions.

Corollary 4.5 Let $\mathcal{D}$ be a staircase diagram with a linear ordering $B_{1}, \ldots, B_{n}$, and let $\mathcal{D}^{i}$ be the subdiagram $\mathcal{D}^{i}:=\left\{B_{1}, \ldots, B_{i-1}\right\}, i=2, \ldots, n$. Then

$$
\Lambda\left(\mathcal{D}^{i+1}\right)=\bar{\lambda}\left(B_{i}\right) \cdot \Lambda\left(\mathcal{D}^{i}\right)
$$

is a $B P$ decomposition with respect to $\mathrm{S}\left(\mathcal{D}^{i}\right)$ for every $i=2, \ldots, n$.

\section{The bijection theorem}

A complete BP decomposition of an element $w \in W$ is a factorization $w=v_{n} \cdots v_{1}$ such that $v_{i}\left(v_{i-1} \cdots v_{1}\right)$ is a Grassmannian BP decomposition for every $i=2, \ldots, n$. We say that $w$ has a complete maximal BP decomposition if each $v_{i}$ is maximal in $W_{S\left(v_{i}\right)}^{S\left(v_{i}\right) \cap J_{i}}$, where $J_{i}=S\left(v_{i-1} \cdots v_{1}\right)$. It is not hard to see that $w$ has a complete maximal BP decomposition if and only if $w=v_{n} \cdots v_{1}$, where $v_{i}\left(v_{i-1} \cdots v_{1}\right)$ is a (not necessarily Grassmannian) BP decomposition with respect to $J_{i}=S\left(v_{i-1} \cdots v_{1}\right)$, and each $v_{i}$ is maximal in $W_{S\left(v_{i}\right)}^{S\left(v_{i}\right) \cap J_{i}}$.

Theorem 5.1 Let $(W, S)$ be any Coxeter group, and let $\Gamma$ be the Coxeter-Dynkin graph. Then the map $\mathcal{D} \mapsto \Lambda(\mathcal{D})$ defines a bijection between spherical staircase diagrams on $\Gamma$ and elements of $W$ with a complete maximal BP decomposition.

By Corollary 4.5, we already know that $\Lambda(\mathcal{D})$ will have a complete maximal BP decomposition. Thus the content of the theorem is that every element with a complete maximal BP decomposition comes from one (and exactly one) staircase diagram. As a corollary, we recover Theorem 1.1 in the simply-laced case:

Corollary 5.2 If $W$ is a simply-laced finite Weyl group with Coxeter-Dynkin graph $\Gamma$, then the map $\mathcal{D} \mapsto$ $\Lambda(\mathcal{D})$ defines a bijection between staircase diagrams $\mathcal{D}$ on $\Gamma$, and smooth elements of $W$.

Proof: Apply Theorems 2.2, 2.3 and 5.1 .

The main challenge in proving Theorem 5.1 is showing that the map is injective. For this, we make the following definition:

Definition 5.3 If $w \in W$, let $\operatorname{bp}(w):=\{s \in S \mid w$ has a BP decomposition with respect to $S \backslash\{s\}\}$. 
If $\mathcal{D}_{1}$ and $\mathcal{D}_{2}$ are two staircase diagrams with $\Lambda\left(\mathcal{D}_{1}\right)=\Lambda\left(\mathcal{D}_{2}\right)$, then obviously bp $\left(\Lambda\left(\mathcal{D}_{1}\right)\right)=\operatorname{bp}\left(\Lambda\left(\mathcal{D}_{2}\right)\right)$. The next theorem shows that $\operatorname{bp}\left(\Lambda\left(\mathcal{D}_{i}\right)\right)$ can be determined from the maximal blocks of $\mathcal{D}_{i}$, making it possible to prove that $\mathcal{D}_{1}=\mathcal{D}_{2}$ inductively.

Theorem 5.4 If $\mathcal{D}$ is a spherical staircase diagram, then

$$
\operatorname{bp}(\Lambda(\mathcal{D}))=\bigcup_{B \in \max (\mathcal{D})} B \backslash J_{R}(B),
$$

where $\max (\mathcal{D})$ is the set of maximal blocks of $\mathcal{D}$.

\section{Sketch of the enumeration}

In this section, we outline the fundamental principals used to enumerate staircase diagrams and prove Theorem 1.2 in the type A case. If $\Gamma$ contains a unique path connecting $t, r \in S$, let $[t, r] \subseteq S$ denote the vertices of this path with endpoints included. We begin with a few important definitions on staircase diagrams.

Definition 6.1 We say $s \in S$ is critical point of $\mathcal{D}$ if $\left|\mathcal{D}_{s}\right|=1$. The collection of critical points is called the critical set of $\mathcal{D}$. Furthermore, we say $\mathcal{D}$ is an elementary diagram if the critical set of $\mathcal{D}$ is contained in the leaves of the support $\mathrm{S}(\mathcal{D})$.

We enumerate staircase diagrams by first decomposing a diagram into elementary diagrams along critical points. For example, we have

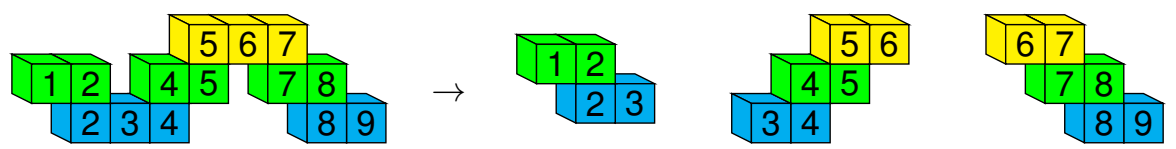

For this reason, we focus on enumerating elementary diagrams. We consider a particular family of graphs and the relationship between their elementary diagrams. Suppose we have a fixed graph $\Gamma$ with distinguished vertex $s \in S$, and vertex set $S$ of size $q$. Define the graph $\Gamma_{q+p}$ to be the graph where we attach a line graph of $p$ vertices to the vertex $s$ and let $S_{n}$ denote the set of vertices in $\Gamma_{n}$. In particular, $\Gamma_{q}=\Gamma$ and for any $n \geq q, \Gamma_{n}$ is a graph with $n$ vertices. Set $s_{q}=s$, and for $n>q$ let $s_{n}$ denote the new leaf in the graph $\Gamma_{n}$. Next, define $Z_{\Gamma}(n)$ to be the set of fully supported elementary diagrams on the graph $\Gamma_{n}$ where $s_{n}$ is a critical point. Define

$$
Z_{\Gamma}^{+}(n):=\left\{\mathcal{D} \in Z_{\Gamma}(n) \mid s_{n} \text { is contained in a maximal block }\right\} .
$$

We define $Z_{\Gamma}^{-}(n)$ similarly by requiring that $s_{n}$ be contained in a minimal block. If $\mathcal{D} \in Z_{\Gamma}^{+}(n)$ then the maximal block $B_{\mathcal{D}}:=\max \left(\mathcal{D}_{s_{n}}\right)$ is unique. The following is an algorithm for constructing elementary diagrams in $Z_{\Gamma}^{+}(n+1)$ from elementary diagrams in $Z_{\Gamma}^{+}(n)$.

Definition 6.2 Let $\mathcal{D} \in Z_{\Gamma}^{+}(n)$ and let $P\left(B_{\mathcal{D}}\right)$ denote the collection of connected, proper, and nonempty subsets of $B_{\mathcal{D}}$ containing $s_{n}$. Define the set of staircase diagrams $\mathfrak{G}_{p}(\mathcal{D}) \subseteq Z_{\Gamma}^{+}(n+p)$ as follows. First define

$$
\mathfrak{G}_{1}(\mathcal{D}):=\left\{\mathcal{D}^{0}\right\} \cup \bigcup_{B^{\prime} \in P\left(B_{\mathcal{D}}\right)}\left\{\mathcal{D}^{B^{\prime}}\right\}
$$


where $\mathcal{D}^{0}:=\left\{B^{0} \mid B \in \mathcal{D}\right\}$ with the same covering relations as $\mathcal{D}$ and

$$
B^{0}:=\left\{\begin{array}{ll}
B & \text { if } B \notin \mathcal{D}_{s_{n-1}} \cup\left\{B_{\mathcal{D}}\right\} \\
B \cup\left\{s_{n}\right\} & \text { if } B \in \mathcal{D}_{s_{n-1}} \\
B \cup\left\{s_{n+1}\right\} & \text { if } B=B_{\mathcal{D}}
\end{array},\right.
$$

and for $B^{\prime} \in P\left(B_{\mathcal{D}}\right)$ we let

$$
\mathcal{D}^{B^{\prime}}:=\mathcal{D} \cup\left\{B^{\prime} \cup\left\{s_{n+1}\right\}\right\}
$$

with the additional covering relation $B_{\mathcal{D}} \prec B^{\prime} \cup\left\{s_{n+1}\right\}$. Recursively define

$$
\mathfrak{G}_{p+1}(\mathcal{D}):=\bigcup_{\mathcal{G} \in \mathfrak{G}_{p}(\mathcal{D})} \mathfrak{G}_{1}(\mathcal{G})
$$

Example 6.3 Let $\Gamma$ be the Dynkin graph of type $A_{3}$ with $s=s_{3}$. Then $\Gamma_{n}$ is the Dynkin graph of type $A_{n}$. If $\mathcal{D}=\left\{\left[s_{1}, s_{2}\right] \prec\left[s_{2}, s_{3}\right]\right\} \in Z_{\Gamma}^{+}(3)$, then $\mathfrak{G}_{p}(\mathcal{D})$ for $p=1,2$ are given by the following diagrams:

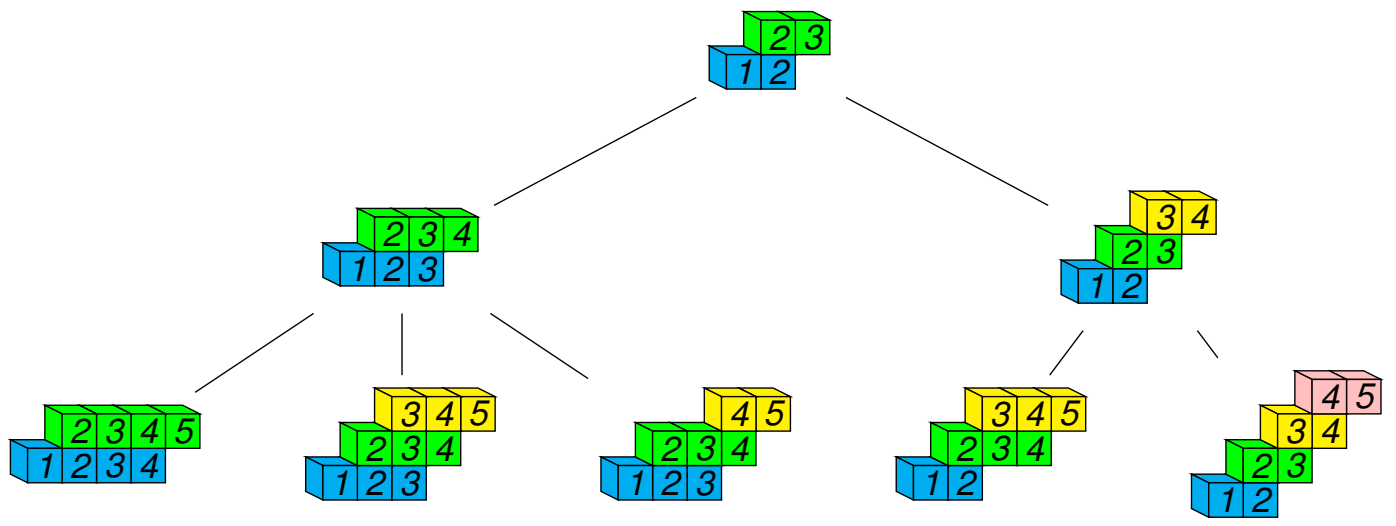

It is easy to see that if $\mathcal{D} \in Z_{\Gamma}^{+}(n)$, then $\mathfrak{G}_{p}(\mathcal{D}) \subseteq Z_{\Gamma}^{+}(n+p)$. The next lemma follows from the definition of elementary diagram and $\mathfrak{G}_{p}$.

Lemma 6.4 If $\mathcal{D}, \mathcal{G} \in Z_{\Gamma}^{+}(n)$ and $\mathcal{D} \neq \mathcal{G}$, then $\mathfrak{G}_{p}(\mathcal{D}) \cap \mathfrak{G}_{p}(\mathcal{G})=\emptyset$ for all $p>0$.

Lemma 6.4 implies that the size of the set $\mathfrak{G}_{p}(\mathcal{D})$ grows predictably. Let $\mathbf{C}_{n}:=\frac{1}{n+1}\left(\begin{array}{c}2 n \\ n\end{array}\right)$ denote the $n$-th Catalan number.

Proposition 6.5 If $\mathcal{D} \in Z_{\Gamma}^{+}(n)$ with $n \geq 3$ and $\left|B_{\mathcal{D}}\right|=2$, then $\left|\mathfrak{G}_{p}(\mathcal{D})\right|=c_{p+1}$.

Proof: Note that $B_{\mathcal{D}}=\left[s_{n-1}, s_{n}\right]$ and hence if $\mathcal{D}^{\prime} \in \mathfrak{G}_{p}(\mathcal{D})$, then $B_{\mathcal{D}^{\prime}}$ is a connected interval contained in $\left[s_{n}, s_{n+p}\right]$. Let $\mathrm{C}_{p, k}$ denote the number of staircase diagrams $\mathcal{D}^{\prime} \in \mathfrak{G}_{p}(\mathcal{D})$ for which $B_{\mathcal{D}^{\prime}}$ is an interval of size $k$. It is easy see from Definition 6.2 that $\mathrm{c}_{p, p+2}=1$ for all $p \geq 0$ and that $\mathrm{c}_{p, p+k}=0$ for all $k \geq 3$. Lemma 6.4 yields the recursion

$$
\mathbf{c}_{p+1, k}=\sum_{i=k-1}^{p+2} \mathbf{c}_{p, i}
$$


and the argument can be finished by summing over $k$.

In Example 6.3, we have that $\left|\mathfrak{G}_{1}(\mathcal{D})\right|=\mathrm{c}_{2}=2$ and $\left|\mathfrak{G}_{2}(\mathcal{D})\right|=\mathrm{c}_{3}=5$.

\subsection{Staircase diagrams of type $A$}

Let $\Gamma$ be the Dynkin graph of type $A_{1}$ with $s=s_{1}$. In the notation of the previous section, $\Gamma_{n}$ is the Dynkin graph of type $A_{n}$.

Proposition 6.6 Let $A_{Z}(t)=\sum_{n=1}^{\infty} z_{n} t^{n}$ be the generating series for elementary staircase diagrams of type $A_{n}$ with full support. Then $A_{Z}(t)=t+t^{2}+2 t^{2}$ Cat $(t)$, where

$$
\operatorname{Cat}(t):=\frac{1-\sqrt{1-4 t}}{2 t}
$$

is the generating series for Catalan numbers.

Proof: It easy to check that $z_{1}=1, z_{2}=3$ and $z_{3}=2$. In particular, $Z_{\Gamma}^{+}(3)$ contains the single staircase diagram $\mathcal{G}:=\left\{\left[s_{1}, s_{2}\right] \prec\left[s_{2}, s_{3}\right]\right\}$. Since the Dynkin diagram of type $A_{n}$ has only two leaves, every elementary staircase diagram is a chain. Consequently, if $n \geq 3$ then the set of elementary staircase diagrams with full support is $Z_{\Gamma}^{+}(n) \cup Z_{\Gamma}^{-}(n)$. In addition, we can check that $Z_{\Gamma}^{+}(n)=\mathfrak{G}_{n-3}(\mathcal{G})$. By Proposition 6.5

$$
A_{Z}(t)=t+3 t^{2}+2 \sum_{n=1}^{\infty} \mathrm{c}_{n} t^{n+2}=t+t^{2}+2 t^{2} \sum_{n=0}^{\infty} \mathrm{c}_{n} t^{n} .
$$

Proposition 6.7 Let $\bar{A}(t):=\sum_{n=1}^{\infty} \bar{a}_{n} t^{n}$ by the generating series for staircase diagrams over $A_{n}$ with full support. Then $\bar{A}(t)=t^{2} /\left(2 t-A_{Z}(t)\right)$.

Proof: Let $\mathcal{D}$ be a staircase diagram of type $A_{n}$ with full support. Let $s_{k}, s_{n}$ be the critical points of $\mathcal{D}$ with the two largest indices. We can write $\mathcal{D}$ as the union of two staircase diagrams $\mathcal{D}^{\prime}, \mathcal{D}^{\prime \prime}$ where we intersect each block of $\mathcal{D}$ with $\left[s_{1}, s_{k}\right]$ and $\left[s_{k}, s_{n}\right]$ respectively. Then $\mathcal{D}^{\prime}$ is a staircase diagram of type $A_{n-k}$ with full support. Furthermore, $\mathcal{D}^{\prime \prime}$ is an elementary diagram with support $\left[s_{k}, s_{n}\right]$. Hence

$$
\bar{a}_{n}=\sum_{k=1}^{n-1} \bar{a}_{k} z_{n+1-k}
$$

This implies that

$$
t^{2}=\sum_{n=1}^{\infty}\left(\bar{a}_{n}-\sum_{k=1}^{n-1} \bar{a}_{k} z_{n+1-k}\right) t^{n+1}=\bar{A}(t)\left(2 t-A_{Z}(t)\right) .
$$


Finally, let $A(t):=\sum_{n=0}^{\infty} a_{n} t^{n}$ where $a_{n}$ denotes the total number of staircase diagrams of type $A_{n}$. Here we set $a_{0}:=1$. Since every staircase diagram is a disjoint union of staircase diagrams with connected support, we conclude:

Theorem 6.8 The generating series $A(t)=\frac{1+\bar{A}(t)}{1-t-t \bar{A}(t)}$.

\section{Acknowledgements}

The authors thank Sara Billey and Jim Carrell for helpful conversations, and Sara Billey for providing data on the number of smooth and rationally smooth Schubert varieties. The first author would like to thank Anthony Kable for some helpful discussions on asymptotics. The second author thanks Mark Haiman for helpful remarks. The pictures of staircase diagrams are based on TikZ code for plane partitions by Jang Soo Kim.

\section{References}

S. Billey and V. Lakshmibai. Singular loci of Schubert varieties, volume 182 of Progress in Mathematics. Birkhäuser Boston, Inc., Boston, MA, 2000. ISBN 0-8176-4092-4. doi: 10.1007/978-1-4612-1324-6. URL http://dx.doi.org/10.1007/978-1-4612-1324-6

S. Billey and A. Postnikov. Smoothness of Schubert varieties via patterns in root subsystems. Adv. in Appl. Math., 34(3):447-466, 2005.

S. C. Billey. Pattern avoidance and rational smoothness of Schubert varieties. Adv. Math., 139(1):141$156,1998$.

M. Bóna. The permutation classes equinumerous to the smooth class. Electron. J. Combin., 5:Research Paper 31, 12 pp. (electronic), 1998.

M. Bousquet-Mélou and S. Butler. Forest-like permutations. Ann. Comb., 11(3-4):335-354, 2007. ISSN 0218-0006. doi: 10.1007/s00026-007-0322-1. URL http://dx.doi.org/10.1007/ s00026-007-0322-1.

J. B. Carrell and J. Kuttler. Smooth points of $T$-stable varieties in $G / B$ and the Peterson map. Invent. Math., 151(2):353-379, 2003. ISSN 0020-9910. doi: 10.1007/s00222-002-0256-5. URL http: //dx.doi.org/10.1007/s00222-002-0256-5.

P. Flajolet and R. Sedgewick. Analytic combinatorics. Cambridge University Press, Cambridge, 2009. ISBN 978-0-521-89806-5. doi: 10.1017/CBO9780511801655. URL http://dx.doi.org/10 . $1017 / \mathrm{CBO} 9780511801655$

M. Haiman. Enumeration of smooth Schubert varieties. preprint. unpublished.

E. Richmond and W. Slofstra. Billey-Postnikov decompositions and the fibre bundle structure of Schubert varieties. to appear in Math. Ann., a. DOI: 10.1007/s00208-015-1299-4.

E. Richmond and W. Slofstra. Staircase diagrams and enumeration of smooth Schubert varieties. preprint, b. arXiv: 1510.06060 . 